\title{
Expression of Shigella flexneri gluQ-rs gene is linked to $d k s A$ and controlled by a transcriptional terminator
}

Valeria C Caballero ${ }^{1,4}$, Viviana P Toledo ${ }^{1}$, Cristian Maturana ${ }^{1,2}$, Carolyn R Fisher $^{3}$, Shelley M Payne ${ }^{3}$ and Juan Carlos Salazar ${ }^{1 *}$

\begin{abstract}
Background: Glutamyl queuosine-tRNA ${ }^{\text {Asp }}$ synthetase (GluQ-RS) is a paralog of the catalytic domain of glutamyl-tRNA synthetase and catalyzes the formation of glutamyl-queuosine on the wobble position of tRNA ${ }^{\text {Asp }}$. Here we analyze the transcription of its gene in Shigella flexneri, where it is found downstream of $d k s A$, which encodes a transcriptional regulator involved in stress responses.

Results: The genomic organization, dksA-gluQ-rs, is conserved in more than 40 bacterial species. RT-PCR assays show co-transcription of both genes without a significant change in transcript levels during growth of $S$. flexneri. However, mRNA levels of the intergenic region changed during growth, increasing at stationary phase, indicating an additional level of control over the expression of gluQ-rs gene. Transcriptional fusions with lacZ as a reporter gene only produced $\beta$-galactosidase activity when the constructs included the $d k s A$ promoter, indicating that gluQ-rs do not have a separate promoter. Using bioinformatics, we identified a putative transcriptional terminator between $d k s A$ and gluQ-rs. Deletion or alteration of the predicted terminator resulted in increased expression of the lacZ reporter compared with cells containing the wild type terminator sequence. Analysis of the phenotype of a gluQ-rs mutant suggested that it may play a role in some stress responses, since growth of the mutant was impaired in the presence of osmolytes.
\end{abstract}

Conclusions: The results presented here, show that the expression of gluQ-rs depends on the $d k s A$ promoter, and strongly suggest the presence and the functionality of a transcriptional terminator regulating its expression. Also, the results indicate a link between glutamyl-queuosine synthesis and stress response in Shigella flexneri.

Keywords: tRNA modification, Gene expression, Stringent response, Osmotic stress

\section{Background}

The fidelity of the translation process depends on the aminoacyl-tRNA synthetase enzymes (aaRS). These essential enzymes are responsible for the correct attachment of the corresponding amino acid onto the cognate tRNA, therefore organisms have at least 20 synthetases [1]. The enzymes are divided in two classes, each class having a conserved structure. The genes encoding the aaRS are easily detected within sequenced genomes $[2,3]$, and some species contain synthetase gene

\footnotetext{
* Correspondence: jcsalazar@med.uchile.cl

'Program of Microbiology and Mycology, Institute of Biomedical Science (ICBM), Faculty of Medicine, University of Chile, Santiago, Chile

Full list of author information is available at the end of the article
}

duplications, such as the glutamyl-tRNA synthetases (GluRS) in Acidithiobacillus ferrooxidans and Helicobacter pylori (genes gltX1 and gltX2) [4,5]. aaRS paralogs, predicted sequences with homology to fragments of synthetases, have also been identified, which is not surprising given the modular nature of the aaRS [6]. Some of the paralogs may be pseudogenes while others have known functions. For instance HisZ from Lactococcus lactis, which has similarity with the catalytic domain of histidyl-tRNA synthetase, is involved in histidine biosynthesis [7]. A recent study in Salmonella enterica has shown that PoxA, encoded by poxA/genX, has similarity to the carboxy-terminal catalytic domain of lysinetRNA synthetase and is required for posttranslational

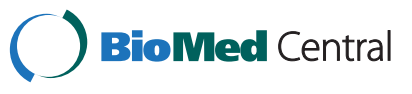


aminoacylation of bacterial elongation factor P. A poxA mutant has reduced colonization and virulence, possibly due to misregulated expression of proteins encoded by the SPI-1 pathogenicity island $[8,9]$.

An Escherichia coli glutamyl-tRNA synthetase paralog, glutamyl queuosine-tRNA ${ }^{\text {Asp }}$ synthetase (GluQ-RS) has approximately $35 \%$ amino acid similarity with the catalytic domain of GluRS. This includes the amino acids involved in recognition and activation of glutamate. Although GluQ-RS is missing the carboxyl-terminus domain responsible for the tRNA recognition, in $E$. coli this enzyme is able to activate the amino acid in the absence of the tRNA. Further, once the aminoacyl-adenylate has been formed, the enzyme attaches the glutamate to the nucleoside queuosine present onto the tRNA ${ }^{\text {Asp }}$. Therefore, this enzyme is involved in the synthesis of a new modified nucleoside glutamyl-queuosine (GluQ) present in tRNA $^{\text {Asp }}[10,11]$. This modification is present in tRNA isolated under acidic conditions from bacterial cells grown in rich media. However, the enzyme is not essential for growth of $E$. coli in rich or minimal media [10]. Queuosine is widely distributed in bacteria, and it is present in the first base of the anticodon of tRNA ${ }^{\text {Asp }}$, tRNA $^{\text {Asn }}, \mathrm{tRNA}^{\text {His }}$ and $\mathrm{tRNA}^{\mathrm{Tyr}}$ [12]; however in E. coli only $\mathrm{RNA}^{\text {Asp }}$ is a substrate for the GluQ-RS enzyme.

The presence of modifications within the anticodon loop of the tRNA, could enhance the accuracy of the codon binding [13]. Then the tRNA ${ }^{\text {AspQ34 }}$ might improve recognition of both GAC and GAU codons [14] and stimulate the binding of the GAU codon to the ribosome [15]. In Shigella flexneri it has been shown that mutations in genes required for tRNA modifications, miaA and tgt decreased virulence. miaA is required for 2-methylthio- $\mathrm{N}^{6}$-isopentenyladenosine modification at position 37 of the anticodon loop and tgt is involved in queuosine modification at position 34 within the anticodon loop [16-18]. In this study, we determined the role of the genome organization and its effect on the expression of the gluQ-rs gene in the major human pathogen, S. flexneri.

\section{Results}

\section{Genomic organization of the S. flexneri gluQ-rs gene}

GluQ-RS is required for the synthesis of the modified nucleoside, GluQ, present on tRNA ${ }^{\text {Asp }}[10,11]$. By searching the bacterial protein database Uniprot (http:// www.uniprot.org/), we were able to identify GluQ-RS in more than a hundred bacterial species, primarily proteobacteria (Figure 1, filled symbols). From the phylogenetic analysis we can distinguished the three subgroups of enzymes described by Dubois et al., 2004 [11], which are characterized by the presence of the signature HXGS, HXGN or HXGH in the adenylate binding site. A similar tree was obtained using the Neighbor joining method.
Phylogenetic analysis within the subgroup of enzymes with the HXGN motif, included representatives from the Firmicutes bacterial group (Figure 1, open square) together with Desulfovibrio vulgaris and Truepera radiovictrix enzymes. From the alignment, these members have 8 characteristic amino acids, G $_{70}$ PDXGGXX, that do not align with the other GluQ-RS (Figure 1, numbering is derived from $D$. vulgaris enzyme). Further genomic analysis indicated that the gluQ-rs gene is found primarily in two genomic arrangements, either alone or located immediately downstream of $d k s A$. Searching within the String database [19] and GenomeNet [20], we found that the $d k s A$-gluQ-rs gene organization was conserved in more than 40 different species, all of which were within the gammaproteobacteria group. These included species of Aeromonadales, Alteromonadales, Enterobacteriaceae, including E. coli and S. flexneri, Pseudomanadales, and Vibrionaceae (Figure 1).

A bioinformatics analysis of the intergenic region between $d k s A$ and gluQ-rs showed great variation in the distance between the two genes among these bacterial species. In $S$. flexneri the intergenic region between the stop codon of $d k s A$ and the first codon of gluQ-rs is only 39 base pairs. Therefore, we suspected that the transcription of gluQ-rs was regulated by the previously characterized $d k s A$ promoter [22]. To test this hypothesis, we isolated total mRNA and performed RT-PCR to identify an mRNA that included both genes (Figure 2A). The observation that there is an mRNA species containing both genes (Figure 2A, lane 1) indicates that they are co-transcribed and that the expression of gluQ-rs may be regulated by the $d k s A$ promoter.

\section{S. flexneri gluQ-rs gene is co-transcribed with $d k s A$ gene}

Although S. flexneri gluQ-rs can be transcribed from the $d k s A$ promoter, this did not rule out the presence of an additional, independent promoter. Therefore, the expression of each gene was measured by RT-PCR during different stages of $S$. flexneri growth in Luria Bertani (LB) at $\mathrm{pH}$ 7.4. The analysis of the $d k s A$ and gluQ-rs transcripts shows that for both mRNAs, the level is stable during the growth curve, with an increase of 1.3-fold at stationary phase compared to the early mid log phase (Figure 2B, compare lanes 2 and 4). However, the mRNA that includes the intergenic region showed variation depending on the stage of growth, increasing 20-fold at stationary phase compared with its expression at early mid log phase (Figure $2 \mathrm{~B} d k s A$ /gluQ-rs, compare lanes 2 and 4). In order to confirm those results, a transcriptional fusion strategy was used. Different segments of the operon were cloned and fused to the lac $Z$ reporter gene in pQF50, and promoter activity was assayed by $\beta$-galactosidase activity [23]. Kang and Craig, 1990 [22] identified three promoters for $d k s A$. By mean of 


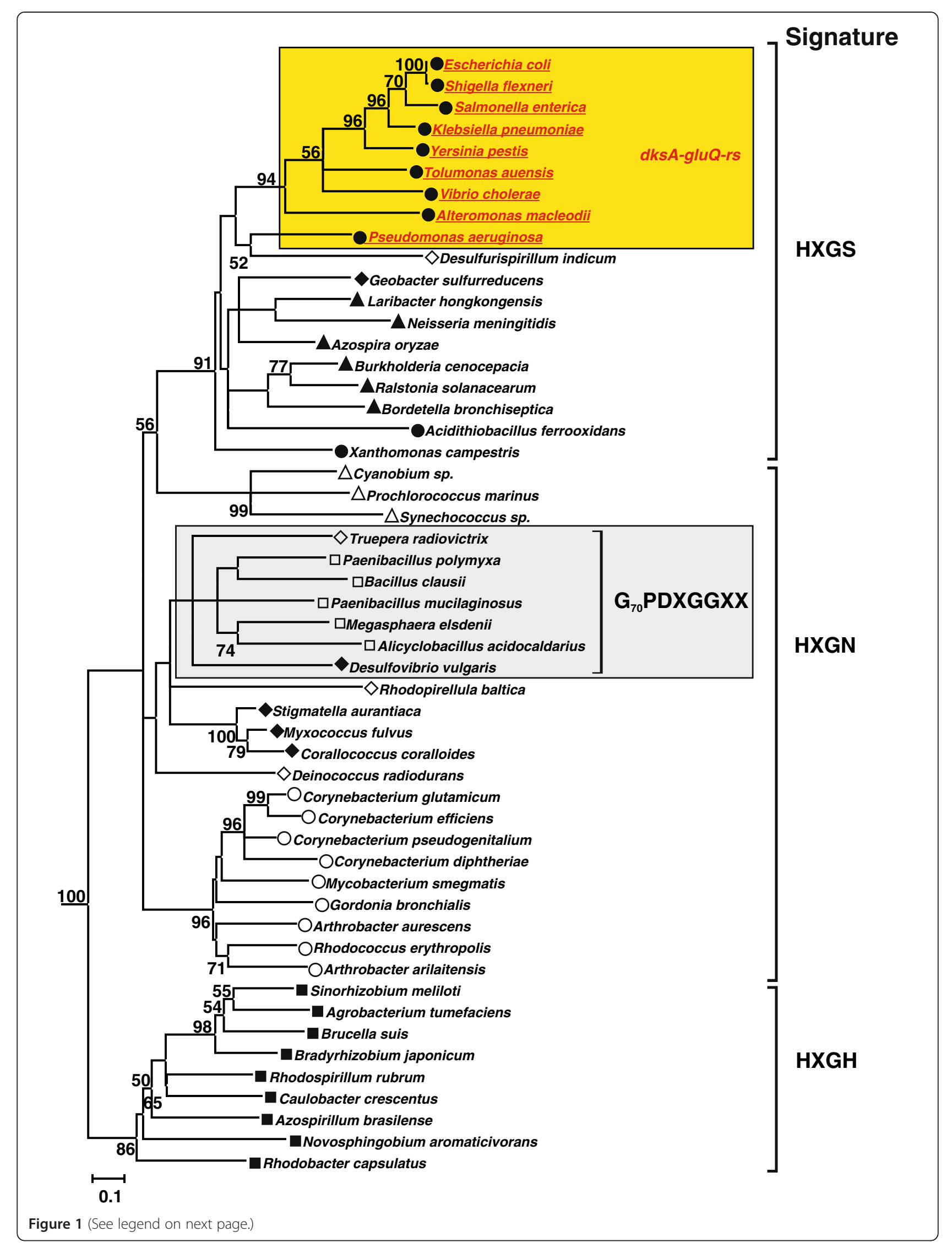


(See figure on previous page.)

Figure 1 GluQ-RS is distributed within the bacterial domain. Rooted Phylogenetic analysis of selected sequences of GluQ-RS, showing the presence of this enzyme in the bacterial domain. Searching within the Uniprot database (http://www.uniprot.org/) of homologues protein to GluQ-RS were identified and then were searched in the GenomeNet (http://www.genome.jp/) database for confirmation and analysis of the genomic organization. Bootstrap values (>50\%) where calculated by 400 replicates using the maximum-likelihood methods in the MEGA5 software [21] and rooted with archaeal GluRS from Methanocaldococcus jannaschii and Archaeoglobus fulgidus (not shown). In yellow background are shown bacterial species (in red and underlined) that are representatives having the genomic organization of $d k s$ A-gluQ-rs genes. The signature of each subgroup identified previously [11] is indicated. Filled symbols representing proteobacteria groups, open symbols represent other bacterial groups. . alphaproteobacteria, $\mathbf{\Lambda}$ : betaproteobacteria, $\bullet$ : gammaproteobacteria, $\bullet$ deltaproteobacteria, 0 : actinobacteria, $\triangle$ : cyanobacteria, $\square$ : firmicutes, $\diamond$ : others.

bioinformatics tools, including BPROM from the Softberry software package (http://linux1.softberry.com/ berry.phtml), we identified those promoters in S. flexneri and included all three promoters in the constructs indicated in Figure 3A. The plasmid containing a fragment from the $d k s A$ promoters to the beginning of the gluQ-rs gene, with the first five amino acids of GluQ-RS, named pVCPDT, represents the full length $d k s A$ gene with its native promoters (Table 1 and Figure $3 \mathrm{~A}$ ). A second fusion construct, $\mathrm{pVCDT}$, contains sequence from the beginning of the coding region of $d k s A$ through the beginning of gluQ-rs and also included the first five amino acids of GluQ-RS. Because pVCDT does not have the $d k s A$ promoter region, it served as the reporter for transcription from an independent gluQ-rs promoter. A third construct, pVCPD, contained the segment from the $d k s A$ promoter to the end of the $d k s A$ gene, hence this plasmid does not have the intergenic region, nor the first amino acids of GluQ-RS (Table 1). Each of the recombinant plasmids was transformed into $S$. flexneri and the $\beta$-galactosidase activity was measured when the bacterial cells reached mid-log phase. Analysis of the enzymatic activity of these reporter fusions showed that the strain carrying pVCDT had baseline levels of the enzyme (Figure $3 \mathrm{~B}$ ), indicating that there is not an independent promoter for $g l u Q-r s$. Thus, the promoter upstream of $d k s A$ is responsible for the expression of both genes, at least under the conditions assayed (see lane pVCPDT Figure 3B). Therefore, these two results (Figure 2 and Figure 3B) indicate that $d k s A$ and gluQ-rs form an operon, and gluQ-rs lacks an additional, separate promoter. A surprising observation was obtained with the clone containing PVCPD, which showed a tenfold increase in enzymatic activity compared to pVCPDT (Figure 3B). This suggested the presence of a terminator or other regulatory sequence in the intergenic region that modulated the expression of $g l u Q-r s$.

The S. flexneri gluQ-rs gene has an upstream transcription terminator

In order to explain the difference observed in expression of lac $Z$ from the recombinant plasmids $\mathrm{pVCPDT}$ and
pVCPD a bioinformatic analysis using mFold [26] was performed to search for possible secondary structures in the mRNA. A potential transcriptional terminator was found at the beginning of the gluQ-rs gene, leaving the first predicted AUG codon located on the bulge of this terminator (Figure 4A). In order to determine the functionality of this terminator, we performed site directed mutagenesis to disrupt the structure in the predicted stem (Figure 4A). As shown in Figure 4B, the plasmid containing the mutations, pVCPDTMut had $>2$-fold higher enzymatic activity $(p<0.05)$ than the plasmid containing the wild type sequence. This result suggested that the intergenic region upstream of gluQ-rs contains a transcriptional terminator.

\section{Identification of the first methionine}

The first methionine in the predicted GluQ-RS protein corresponds to the one located on the bulge of the terminator structure (Figure 4A), which also contains a possible Shine-Dalgarno sequence. However, in related species like Escherichia fergusonii that also have the terminator structure, a methionine is not present at that location. In the $S$. flexneri sequence, there is another AUG codon in the same reading frame 27 nucleotides downstream from the one in the terminator. In order to determine which methionine is the start site for translation of the S. flexneri GluQ-RS, we constructed a vector that included the intergenic region from the stop codon of the $d k s A$ gene to the end of gluQ-rs cloned into the expression vector $\mathrm{pET} 15 \mathrm{c}$. This allowed expression of C-terminal His-tagged GluQ-RS under T7 promoter control. The protein was partially purified by affinity chromatography as described elsewhere [10], and the sequence of the amino terminus of the GluQ-RS protein was determined to be $\mathrm{NH}_{2}$-T-D-T-Q-Y-I-G-RF-A-P, which corresponds to the amino acid sequence after the second methionine. Therefore, the initiator methionine is not the one indicated in the database, and the protein is 298 amino acids. Surprisingly, there is no obvious Shine-Dalgarno sequence adjacent to the initiator methionine we identified (Figure 5). 
A

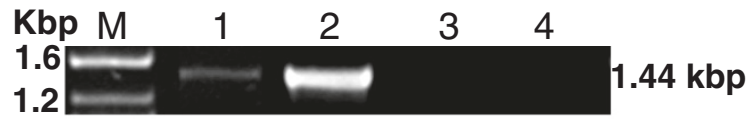

B $\frac{\text { cDNA }}{1234}+\begin{aligned} & \text { RNA } \\ & \frac{1}{1} 324\end{aligned}$

$d k s A=-\infty-\infty 36$ bp
381240275326

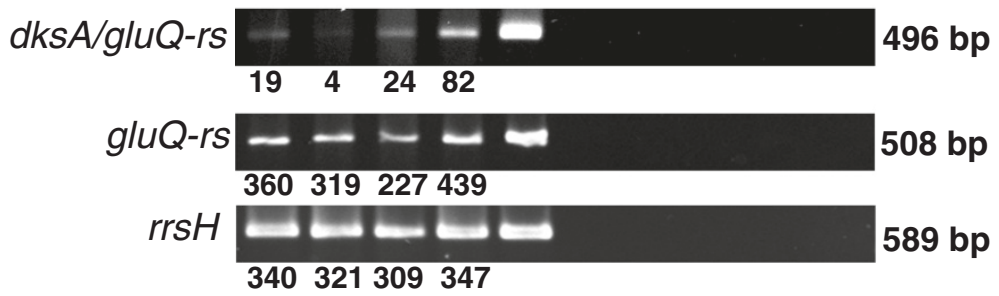

Figure 2 gluQ-rs is co-transcribed with $d \mathbf{k s} A$ in S. flexneri 2457T. A) Agarose gel showing the amplified product of the full-length operon extending from the $d k s A$ gene through the end of gluQ-rs (1.44 kpb). Total RNA isolated during mid log phase growth of $S$. flexneri was subjected to reverse transcriptase and PCR (RT-PCR) using primers opeF/opeR (Table 2). M: molecular marker, sizes are indicated. Lane 1: RNA treated with reverse transcriptase, Lane 2: genomic DNA isolated from S. flexneri 2457T, Lane 3: RNA without reverse transcriptase treatment, Lane 4: negative control of PCR reaction without DNA. B) Electrophoretic analysis of each amplified gene fragment, dksA (dksAF/dksAR; 436 bp), g/uQ-rs (gQRSF/ gQRSR; 508 bp), the intergenic region dksAvgluQ-rs (interF/interR; 496 bp) and the ribosomal RNA 165 (rrsHF/rrsHR, 589 bp). Total RNA isolated during different phases of the growth curve of S. flexneri $2457 \mathrm{~T}$ was subjected to RT-PCR to detect the corresponding fragment. Lane 1: lag phase, Lane 2: early mid log phase, Lane 3 : mid log phase, Lane 4 : stationary phase. + : corresponds to amplification using genomic DNA. RNA: Isolated RNA without reverse transcriptase treatment. -: negative control PCR reaction without DNA. Each band was estimated using Image J software (V1.46) and its amount was estimated using the fragment corresponding to $500 \mathrm{bp}$ of the DNA ladder.

\section{Phenotype of the S. flexneri gluQ-rs mutant}

To determine the role of GluQ-RS in S. flexneri growth and virulence, a deletion mutant of the gluQ-rs gene was constructed in S. flexneri 2457T. The mutant was compared to the wild type by Biolog phenotype MicroArrays (Biolog, Inc., Almeda, CA). The major difference observed for the mutant was impaired metabolism when grown under osmotic stress conditions (Figure 6). The mutant had a longer lag and reduced growth compared to the wild type in the presence of increasing concentrations of potassium chloride, sodium sulfate, sodium formate, sodium benzoate, sodium nitrate and sodium nitrite. The phenotype was complemented with the gluQ$r s$ gene cloned into an expression vector. No differences were observed in the growth or metabolism of these strains when they were incubated in presence of $1 \%$ sodium chloride, which was similar to LB (Figure 6 and data not shown).

Because expression of $d k s A$ is required for $S$. flexneri virulence [27], and growth of Shigella in the intracellular environment may induce a stress response, we also measured invasion and plaque formation by the gluQ-rs mutant. However, no significant differences were noted (data not shown), suggesting that GluQ-RS is not essential for invasion or intracellular growth of S. flexneri.

\section{Discussion}

Conserved dksA-gluQ-rs genomic organization in gammaproteobacteria

GluQ-RS, a paralog of GluRS synthetase, is involved in the formation of GluQ, the nucleoside located at the wobble position of $\mathrm{tRNA}^{\mathrm{Asp}}$ in bacteria. The protein is present in Firmicutes, Actinobacteria, Cyanobacteria, Alphaproteobacteria, Betaproteobacteria, Gammaproteobacteria and Deltaproteobacteria (Figure 1). From the phylogenetic analysis we distinguished the three subgroups described previously [11] based on the HIGH motif that is present in the class I aminoacyl-tRNA synthetases [2]. As was described previously [11], all GluQ-RS enzymes are characterized by the replacement of a threonine in GluRS enzymes, which is involved in the recognition of the amino acid and the terminal adenosine of the tRNA ${ }^{\text {Glu }}\left(\mathrm{Thr}_{133}\right.$ of Methanocaldococcus jannaschii GluRS enzyme) by isoleucine, leucine or valine at that position ( $\mathrm{Ile}_{47}$ of $S$. flexneri GluQ-RS). This substitution is also conserved in all enzymes analyzed here, including those from the Firmicutes group.

The gluQ-rs gene is widely distributed in the bacterial domain; however, its genome organization is variable. We observed that only in members of the gammaproteobacteria, namely Aeromonadales, Alteromonadales, Pseudomonadaceae, 

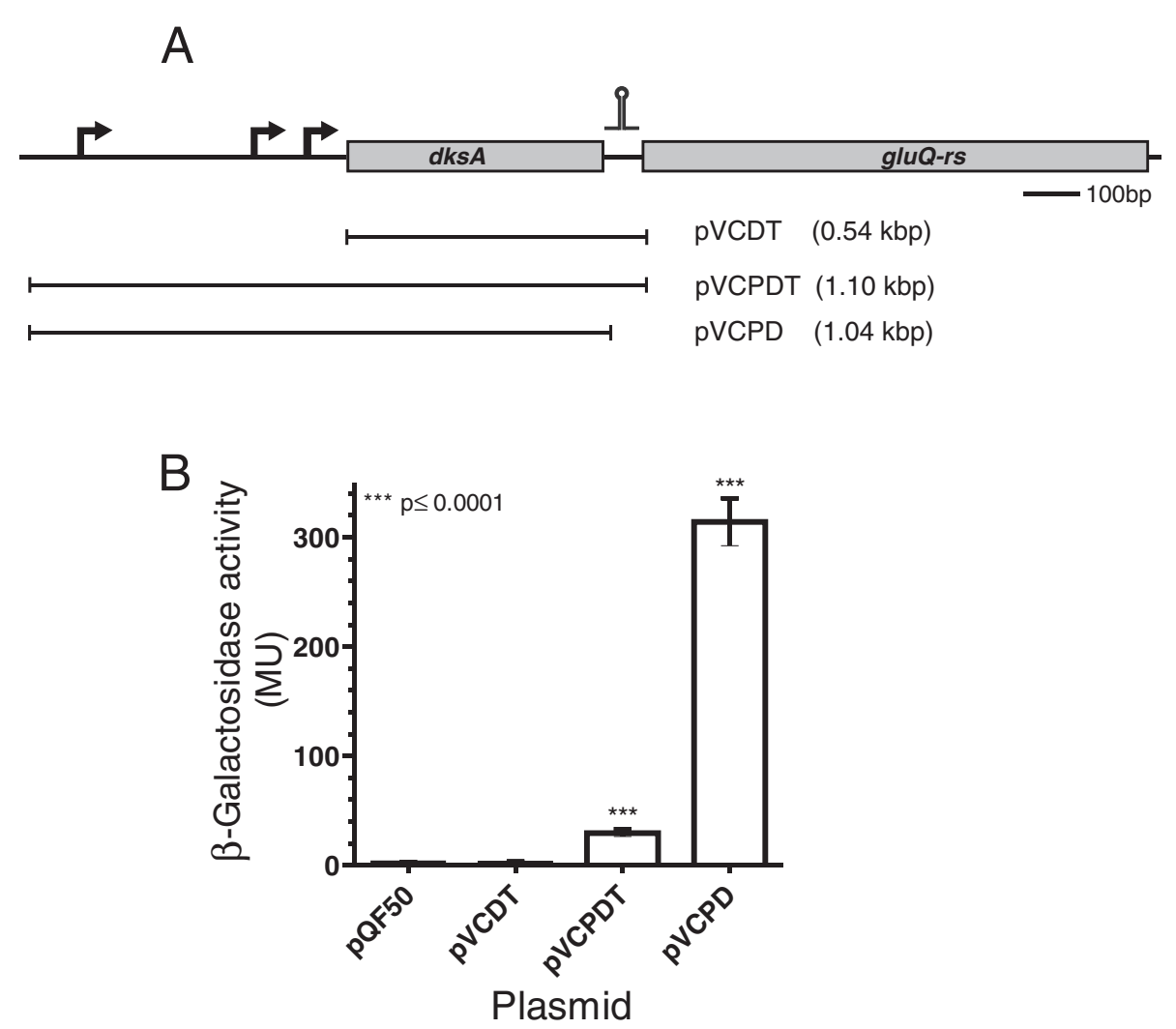

Figure 3 The transcription of the gluQ-rs gene is controlled by a termination stem loop. A) Schematic representation of the operon, the arrows indicate the position of each promoter identified by our bioinformatics analysis and experimentally determined by Kang and Craig, 1990 [22]. The putative $\rho$-independent terminator is represented by the stem loop symbol upstream of gluQ-rs gene. The horizontal bar represents the DNA region amplified and cloned into pQF50 (Table 1). The recombinant plasmids are described in Table 1. pVCDT does not have the $d k s A$ promoter but has the terminator. pVCPDT has the promoter region of $d k s A$ and the terminator upstream of gluQ-rs; therefore, it represents the genomic organization of the operon. pVCPD also has the promoter of $d k s A$ but lacks the terminator region. The size of each fragment is indicated. B) $\beta$-galactosidase activity of each protein extract obtained from the corresponding clone. The data represent the average of three experiments in triplicates and the Student $t$ test was used to compare the means between each clone with the empty vector. ${ }^{* * *} p$ values $<0.05$ were considered statistically significant.

Enterobacteriaceae and Vibrionaceae, the gluQ-rs gene is located immediately downstream of the $d k s A$ gene (Figure 1 ). A more detailed analysis shows that even within this genomic organization there are differences. In some species of Pseudomonadaceae, such as $P$. aeruginosa, $P$. entomophila, and $P$. fluorescens, we observed the same genomic structure as in $E$. coli or S. flexneri, with a distinctive terminator between the genes. In contrast, while the $d k s A$ gene is also upstream of gluQ-rs in some P. syringae, there are insertions of an encoded transposase or more than a 400 base pairs separating both genes without a detectable terminator. However, using bioinformatics tools we detected a possible promoter within this region in $P$. syringae (data not shown), indicating that the expression of the gluQ-rs gene may be under control of its own promoter, a question that remains to be addressed.

\section{Stringent response and tRNA modification}

Our bioinformatic analysis shows the presence of a transcriptional terminator and lack of a gluQ-rs-specific promoter. This is consistent with our results, in which we did not detect any activity from promoters other than those upstream of the $d k s A$ gene (Figure 3 ). This unusual arrangement suggests that gluQ-rs expression is dependent on $d k s$ A-regulated conditions. Because DksA is a key member of the stringent response in bacteria and regulates a number of processes in the cell, including its own expression [25,28], the data suggest that there is coordinate regulation of tRNA modification and other DksA targets. Although we could not detect any promoter activity specific for gluQ-rs in the growth conditions tested (i.e. altering the $\mathrm{pH}$, presence of glutamate), we cannot discount the possibility that the gene is specifically regulated under some other conditions. The regulon database (http://regulondb.ccg.unam.mx/index.jsp) indicates that the $E$. coli gluQ-rs gene has a recognition site for the $\sigma^{24}$ subunit of RNA polymerase. From our analysis, this sequence is identical to S. flexneri, but there is no experimental evidence of this recognition. Interestingly, when the gluQ-rs gene was 
Table 1 Bacterial strains and plasmids used in this work

\begin{tabular}{|c|c|c|}
\hline Bacterial strains or plasmid & Characteristics & Source or reference \\
\hline \multicolumn{3}{|l|}{ Shigella flexneri } \\
\hline S. flexneri $2457 \mathrm{~T}$ & Wild type strain & Laboratory stock \\
\hline S. flexneri 2457T $\Delta$ gluQ-rs::kan & Deletion mutant of gluQ-rs gene & This work \\
\hline \multicolumn{3}{|l|}{ Escherichia coli } \\
\hline E. coli W3110 $\Delta$ gluQ-rs::kan & Deletion mutant of gluQ-rs gene & [10] \\
\hline $\mathrm{DH} 5 \mathrm{a}$ & $\begin{array}{l}\text { F } 980 \text { lacZDM15 } \Delta \text { (lacZYA-argF) U169 recA1 endA1 hsdR17 (rK-, mK+) } \\
\text { phoA supE44 } \lambda \text {-thi-1 gyrA relA1 }\end{array}$ & [24] \\
\hline BL21(DE3) & $\begin{array}{l}\text { F ompT gal dcm lon hsdS }\left(r_{B}^{-} m_{B}^{-}\right) \lambda(D E 3 \text { [lacl lacUV5-T7 gene } 1 \text { ind } 1 \\
\text { sam7 nin5]) }\end{array}$ & Invitrogen \\
\hline \multicolumn{3}{|l|}{ Plasmids } \\
\hline $\mathrm{pTZ57R/T}$ & bla, pMB1 ori, lacZ peptide, f1 phage ori & Fermentas $^{\circledR}$ \\
\hline pQF50 & bla, pMB1 ori, lacZ gene without promoter & [23] \\
\hline pET15c & Empty vector, a modified version of pET15b & This work \\
\hline pVCDT & $\begin{array}{l}\text { S. flexneri fragment from nucleotide }+58^{\mathrm{a}} \text { of } d k s \text { a gene to beginning } \\
\text { of gluQ-rs gene }(+590) \text { cloned into pQF50. Pair of primers used were } \\
\text { PgluQF/PdksARCT. }\end{array}$ & This work \\
\hline pVCPDT & $\begin{array}{l}\text { S. flexneri fragment from nucleotide }-506 \text { of } d k s \text { g gene to beginning } \\
\text { of gluQ-rs gene }(+590) \text { cloned into pQF50. Pair of primers used were } \\
\text { PdksAF/PdksARCT. }\end{array}$ & This work \\
\hline pVCPDTMut & $\begin{array}{l}\text { S. flexneri fragment from nucleotide }-506 \text { of } d k s \text { gene to beginning } \\
\text { of gluQ-rs gene }(+590) \text { cloned into pQF } 50 \text {, with the terminator mutated } \\
\text { by the nucleotides indicated in Figure } 4 a \text {. }\end{array}$ & This work \\
\hline pVCPD & $\begin{array}{l}\text { S. flexneri fragment from nucleotide }-506 \text { of } d k s A \text { gene to } \\
\text { nucleotide }+527 \text { (end of } d k s A \text { gene) cloned into pQF50. Pair of primers used } \\
\text { were PdksAF/PdksARST. }\end{array}$ & This work \\
\hline pATGGQRS & $\begin{array}{l}\text { S. flexneri gene from nucleotide }+509 \text { (stop codon of } d k s \text { A) to } \\
\text { nucleotide }+1469 \text { (last codon of gluQ-rs without stop codon). Pair of primers used } \\
\text { were ATGGQRSF/ATGGQRSR. }\end{array}$ & This work \\
\hline
\end{tabular}

${ }^{a}$ Fragments cloned are numbered based on the transcription start of $d k s A$ identified by [25].

deleted in S. flexneri, the mutant showed impaired growth in the presence of osmolytes (Figure 6). A recent publication demonstrated that $\sigma^{24}$ and $\sigma^{\mathrm{S}}$ proteins from Salmonella enterica serovar Typhi are important for the expression of several genes induced by osmotic stress in this bacterium [29]. Moreover, the expression of the gene encoding $\sigma^{24}$ in $E$. coli is regulated by the stringent response [30]. The possible role of $\sigma^{24}$ on the expression of gluQ-rs under osmotic stress might be interesting to study.

GluQ-RS is an enzyme responsible for the formation of the GluQ tRNA modification, and two independent groups $[10,11]$ have shown that this enzyme required a high concentration of glutamate to be activated and transferred to the queuosine base present on the tRNA $^{\text {Asp }}$. Interestingly, one of the first events to occur when bacteria are subject to high osmolyte stress is an increase in glutamate levels within the cytoplasm [31]. Our observation indicates an important role of the tRNA modification for the growth of S. flexneri in the presence of osmolytes (Figure 6). Other tRNA modifications might play a similar role in this stress condition. In $E$. coli, inactivation of the $y f i C$ gene, responsible for the modification at the adenosine 37 present on the tRNA ${ }^{\mathrm{Val}}$, leads to a high sensitivity to osmotic stress [32].

\section{Transcription of gluQ-rs is regulated by a terminator}

The results obtained in the present work show the presence of a terminator and suggested the functionality of this structure (Figure 3 and Figure 4). To our knowledge, there are few examples of bacterial genes that have similar structures. There is a terminator structure upstream of the DNA primase gene, dnaG, which also has an unusual Shine Dalgarno sequence [33]. Another example is the $r e c X$ gene in $E$. coli, where readthrough accounts for approximately $10 \%$ of its transcription [34].

The two characteristics of gluQ-rs described in this work, co-transcription with the upstream gene and the presence of a terminator immediately upstream, allow us to propose that both the transcription and translation process could be regulated in the gluQ-rs gene. It has been described, that the presence of terminators upstream of the coding region might be part of a regulatory system such as a riboswitch [35]. Riboswitches for 


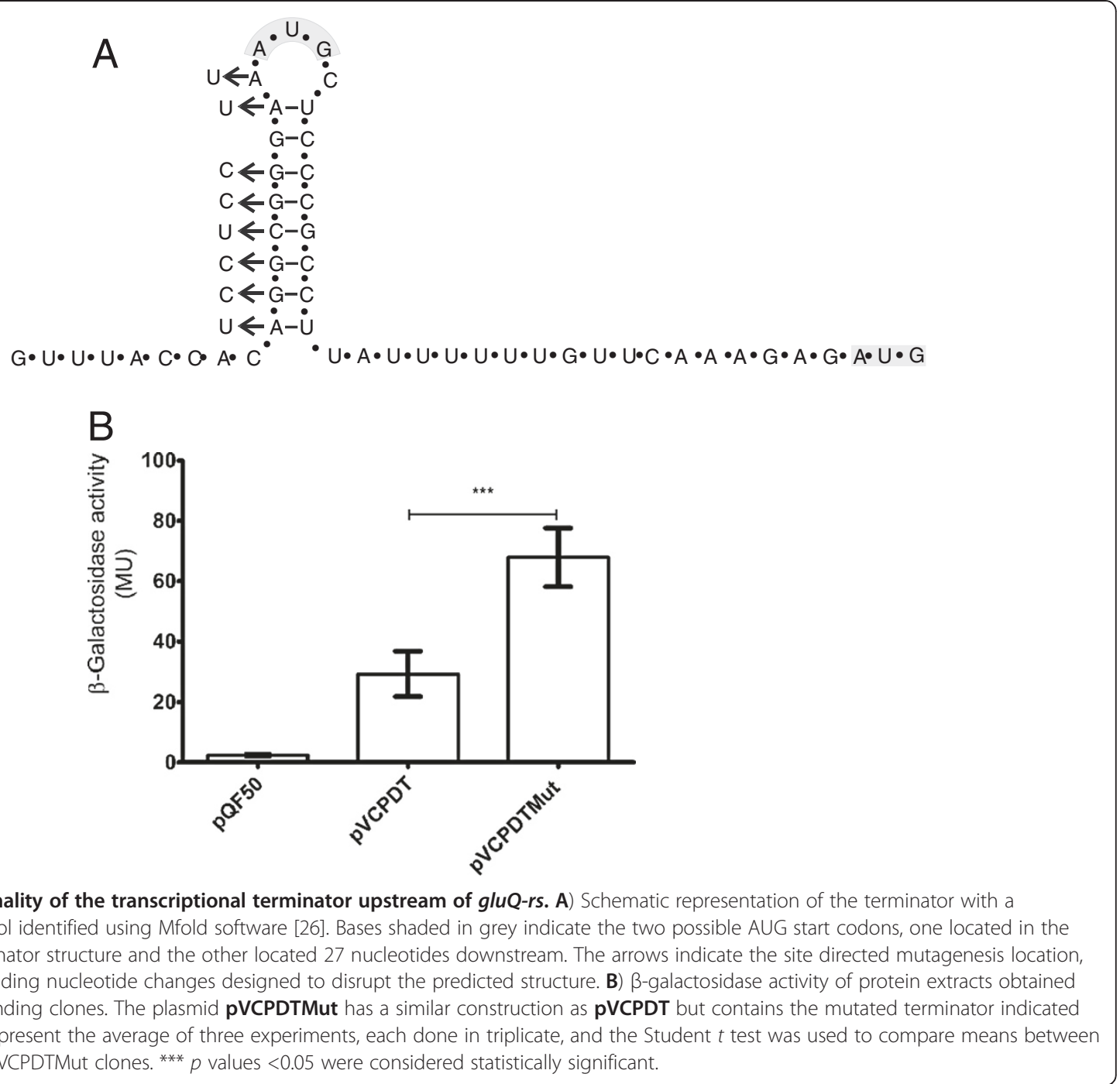

genes involved in queuosine formation have been described, in which the precursor preQ1 is the ligand of the mRNA structure [36]. Using the riboswitch server (ribosw.mbc.nctu.edu.tw [37]), we did not identify any potential riboswitch (data not shown). However we cannot discount that the terminator described here might be part of a regulatory circuit similar to a riboswitch, or that an unidentified protein might bind the terminator structure.

\section{GluQ modification and codon bias}

tRNA modifications present at the anticodon loop might be important for the accuracy of codon reading during the translation processes [13]. Morris et al., 1999 [14] proposed, based on molecular modeling, that the

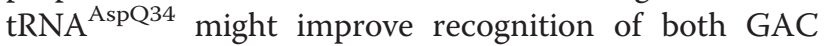
and GAU codons, consequently the interaction of the codon GAU with the anticodon of tRNA ${ }^{\text {AspG34 }}$ could be less efficient. In fact, in $S$. flexneri there are a few genes such as sitA, virF and proX (an inducible gene under osmotic stress) that have a bias toward those codons that favor the modified tRNA. Thus, while there is no obvious loss of plaque formation in the gluQ-rs mutant (data not shown), the absence of GluQ-RS may influence the expression of proteins such as SitA that are required for fitness of Shigella in the host [38].

\section{Conclusions}

In this work we have shown that the expression of $g l u Q-r s$, a gene codifying an enzyme involved in the formation of GluQ present on the tRNA ${ }^{\text {Asp }}$, is under the control of the $d k s A$ promoter. Also, we show the presence of a functional terminator that controls the expression of gluQ-rs. Finally, we present data that suggest that the presence of modification of the tRNA ${ }^{\text {Asp }}$ is important for survival of the human pathogen Shigella flexneri under osmotic stress conditions. 


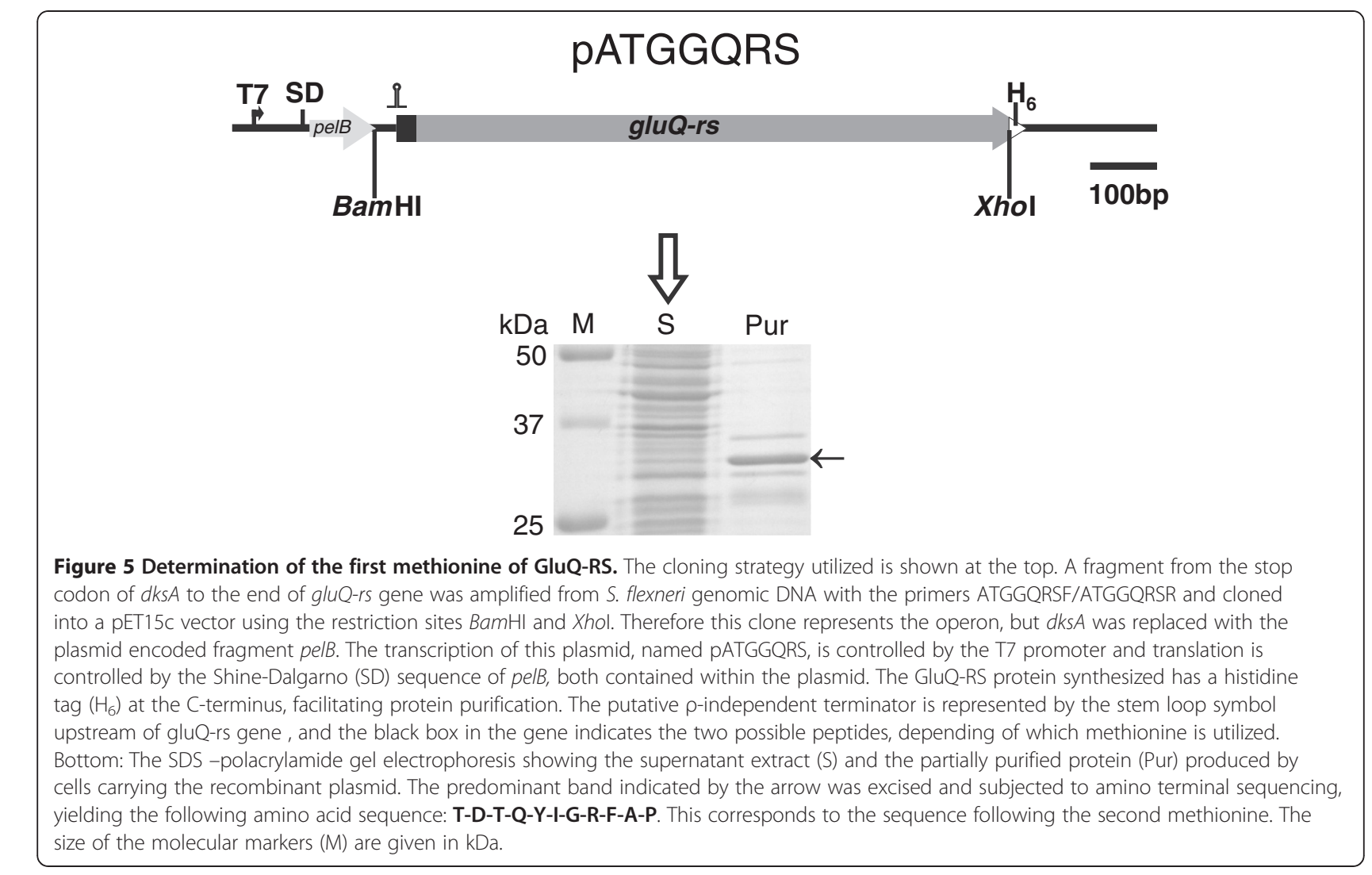

\section{Methods}

Bacterial growth conditions

The bacterial strains and plasmids used in this study are described in Table 1. E. coli strains were maintained on LBagar (10 g of tryptone per liter, $5 \mathrm{~g}$ of yeast extract per liter, $10 \mathrm{~g}$ of $\mathrm{NaCl}$ per liter and $15 \mathrm{~g}$ of agar per liter), Shigella strains were maintained on Trypticase Soy Agar plus 0.01\% congo red. All strains were stored at $-80^{\circ} \mathrm{C}$ in $\mathrm{LB}$ broth plus $20 \%$ glycerol. The bacteria were grown in LB broth adjusted to $\mathrm{pH} 7.4$ with $40 \mathrm{mM}$ MOPS (Merck) or M9 minimal media [24]. When necessary, ampicillin was added to a final concentration of $100 \mu \mathrm{g} / \mathrm{ml}$. Bacterial growth was monitored by optical density at $600 \mathrm{~nm}\left(\mathrm{OD}_{600}\right)$.

\section{Bioinformatics tools to construct the phylogenetic tree}

The protein sequences were obtained from the Uniprot database (http://www.uniprot.org/) and then were searched in the GenomeNet (http://www.genome.jp/) to confirm the

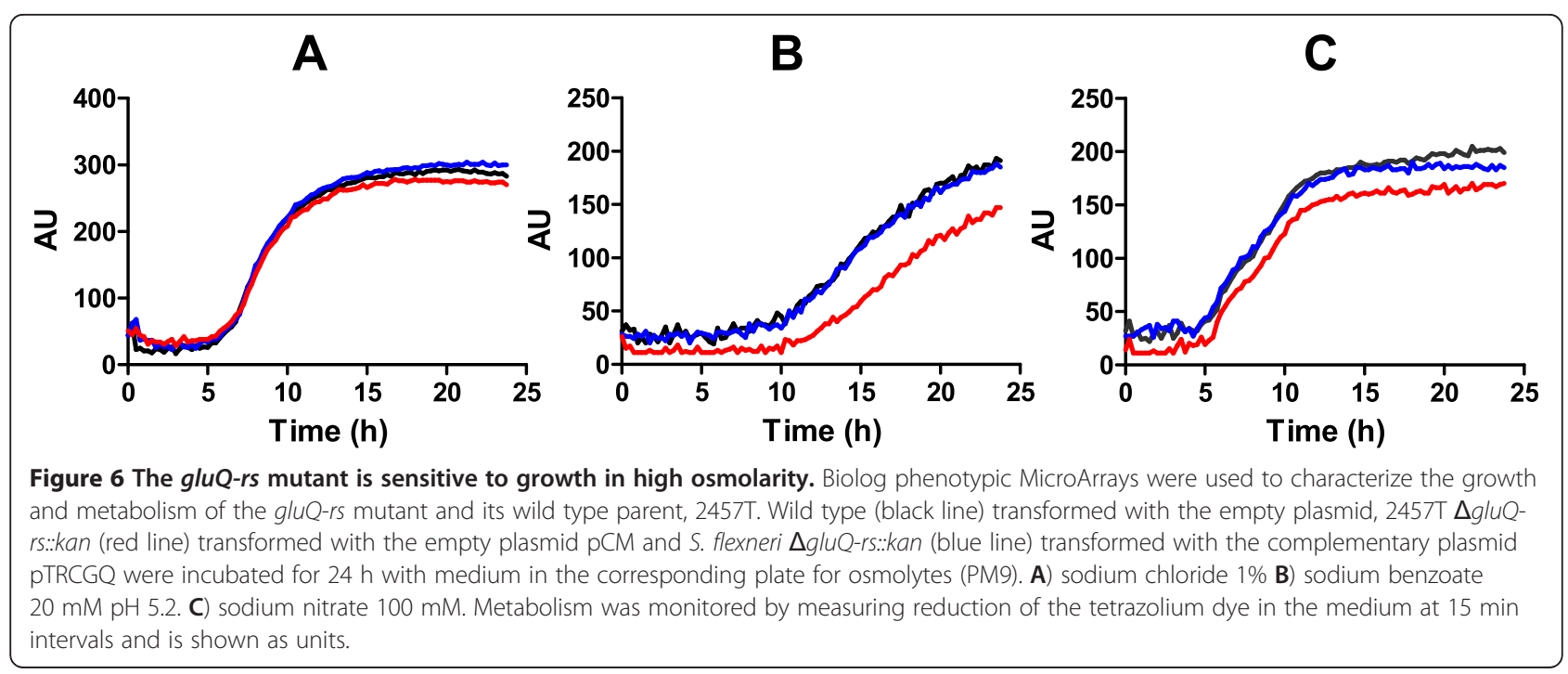


genomic organization. A selected number of GluQ-RS enzymes were aligned using the MUSCLE algorithm [39] and analyzed using the maximum-likelihood method based on the JTT matrix-based model. The percentage of trees in which the associated proteins clustered together is shown next to the branches. The analysis involved 54 amino acid sequences, including the GluRS proteins from Methanocaldococcus jannaschii and Archaeoglobus fulgidus as an outgroup. All positions containing gaps and missing data were eliminated. There were a total of 199 positions in the final dataset. Evolutionary analyses were conducted in MEGA5 [21].

\section{RNA isolation and synthesis of CDNA}

Total mRNA was obtained during the growth of $S$. flexneri $2457 \mathrm{~T}$ using the RNeasy mini kit following the supplier instructions (Qiagen). The purified nucleic acid was treated with RNase- free DNase (Fermentas) and its concentration was estimated by measuring the optical density at $260 \mathrm{~nm}\left(\mathrm{OD}_{260}\right)$. Approximately $1 \mu \mathrm{g}$ of total RNA was subjected to reverse transcription using M-MuLV polymerase (Fermentas) and random primers following the provider's protocol. The cDNA was amplified using specific PCR primers for each gene of interest (Table 2).

\section{Construction of transcriptional fusions}

Transcriptional fusions were constructed to study the expression control of gluQ-rs. Fragments of the $d k s A-g l u Q-r s$ region were fused to $l a c Z$ in the vector $\mathrm{pQF} 50$ by using the BamHI and HindIII restriction sites [23]. Each fragment was amplified from S. flexneri genomic DNA using the indicated primers (Tables 1 and 2) with the High Fidelity PCR Enzyme Mix polymerase (Fermentas) and cloned into pQF50 (Table 1). Once the sequence of each clone was confirmed, the recombinant plasmid was introduced into $S$. flexneri $2457 \mathrm{~T}$ by electroporation. The nomenclature of the recombinants plasmids is: $\mathrm{P}$ for promoter of the $d k s A$ gene, $\mathrm{D}$ for the $d k s A$ gene and $\mathrm{T}$ for a terminator structure.

\section{$\beta$-galactosidase activity}

S. flexneri transformed with the corresponding constructs were cultured overnight in LB, a 1:50 dilution was inoculated into $10 \mathrm{ml}$ culture of $\mathrm{LB} \mathrm{pH} 7.4$ and grown to an $\mathrm{OD}_{600}$ of 0.5 . Aliquots of $0.5 \mathrm{ml}$ of each strain containing the clone or the empty vector were

Table 2 Primer sequences

\begin{tabular}{|c|c|c|}
\hline Name & Sequence $5^{\prime}-3^{\prime a}$ & Reference and characteristics \\
\hline opeF & TAAGGAGAAGCAACATGCAAGA & This work. RT-PCR of $d k s A$ operon from nucleotide +40 to $+1477^{b}$ \\
\hline opeR & ATAGCTCAGCATGACGCATTT & \\
\hline dksAF & ATGCAAGAAGGGCAAAACCG & This work. RT-PCR of $d k s A$ gene from nucleotide +54 to +488 \\
\hline dksAR & GCGAATTTCAGCCAGCGTTI & \\
\hline interF & AGTGGAAGACGAAGATTTCG & This work, RT-PCR of intergenic region from nucleotide +368 to +863 \\
\hline interR & TCCTTGTTCATGTAACCAGG & \\
\hline gQRSF & TTCAAAGAGATGACAGACACACAG & This work, RT-PCR of gluQ-rs gene from nucleotide +567 to +1074 \\
\hline gQRSR & CACGGCGATGAATGATAAAATC & \\
\hline rrsHF & CCTACGGGAGGCAGCAG & [40] RT-PCR of ribosomal RNA $16 \mathrm{~S}$ \\
\hline $\operatorname{rrsHR}$ & CCCCCGTCAATTCCTTTGAGTTI & \\
\hline pcnBR & GATGGAGCCGAAAATGTTGT & Reverse of $p c n B$ gene from nucleotide +1993 \\
\hline PdksAF & GGATCCAAGCGAAGTAAAATACGG & BamHI site, from nucleotide -506 \\
\hline PdksARST & AAGCTTGTGATGGAACGGCTGTAAT & HindIII site, to nucleotide +527 \\
\hline PdksARCT & AAGCTTCTGTGTGTCTGTCATCTCTTTG & Hindlll site, to nucleotide +590 \\
\hline PgluQF & GGATCCAAGAAGGGCAAAACCGTA & BamHI site, from nucleotide +58 \\
\hline TERGQ2 & CCTTATTITTGTTCAAAGAGATGACAGACACACAGA & Recognition from nucleotide +555 \\
\hline TERMGQ3 & ATAAGGCGGGAGCATAACGGAGGAGTGGTAAAC & Recognition from nucleotide +560 , underline sequence are nucleotides changed \\
\hline M13R & GCGGATAACAATTTCACACAGG & Recognition site in $\mathrm{pTZ} 57 \mathrm{R} / \mathrm{T}$ \\
\hline ATGGQRSF & GGATCCGTAATTACAGCCGTTCCATC & $\begin{array}{l}\text { BamHI site, from nucleotide }+507 \text {. Underline nucleotides correspond to the stop } \\
\text { codon of } d k s A\end{array}$ \\
\hline ATGGQRSR & CTCGAGGCATGACGCATTTGAGAATG & Xhol site, to nucleotide +1469 \\
\hline virFF & AGCTCAGGCAATGAAACTTTGAC & [41] \\
\hline $\operatorname{virFR}$ & TGGGCTTGATATTCCGATAAGTC & \\
\hline
\end{tabular}

${ }^{a}$ Nucleotides in bold are indicated restriction site.

${ }^{\mathrm{b}}$ Fragments cloned are indicated based on the transcription start of $d k s A$ identified by [25]. 
assayed for $\beta$-galactosidase activity according to Miller [42]. The data were analyzed using the software GraphPad Prism V5.01.

\section{Site directed mutagenesis}

A possible transcription terminator between $d k s A$ and gluQ-rs was identified using the program Mfold [26]. Site directed mutagenesis by overlap PCR was performed to disrupt the predicted terminator [43]. Using the fragment VCPDT cloned in the vector pTZ57R/T as template, was amplified a 1,072 bp fragment, which include the mutation, using the primers PdksAF and TERMGQ3, while a second fragment of 162 bp overlapping the mutated region, was obtained with primers TERGQ2 and M13R (Table 2). Both fragments (1,072 bp and $162 \mathrm{bp}$ ) were digested with $D p n \mathrm{I}$, purified and mixed at equimolar quantities to carry out a PCR reaction using the $5^{\prime}$ and $3^{\prime}$ ends primers (PdksAF and PdksARCT). The 1,110 bp amplified fragment was cloned in the vector $\mathrm{pTZ} 57 \mathrm{R} / \mathrm{T}$ and sequenced to verify the mutation. This plasmid was digested with BamHI and HindIII and the fragment subcloned in to the vector pQF50.

\section{Determination of first methionine of GluQ-RS}

In order to establish which is the first AUG codon of gluQ-rs, the recombinant plasmid pATGGQRS was constructed. A PCR reaction was performed using the primers ATGGQRSF and ATGGQRSR (Table 2) and genomic DNA from S. flexneri. The amplified fragment, containing the BamHI site, stop codon of $d k s A$, the intergenic region with the terminator, the gluQ-rs reading frame without its stop codon and the XhoI site was cloned into pET15c, a modified version of pET15b, which was constructed by inserting the $290 \mathrm{bp} \mathrm{XbaI}$ and BlpI fragment of pET20b containing the polylinker into pET15b. This construct allowed the synthesis of a Cterminal histidine tagged protein under the transcription control of the T7 promoter. The construct was transformed in BL21(DE3) strain and the His-tagged protein was partially purified by affinity chromatography as described previously [10]. The eluted protein was transferred to a PVDF membrane and stained with Coomassie blue. The predominant band of the expected size (34.6 kDa) was sequenced at the Protein Core Facility of the Institute for Cellular and Molecular Biology, University of Texas at Austin.

\section{Construction of the plasmid for complementation of the gluQ-rs mutation}

This plasmid was constructed from the pATGGQRS plasmid in which the T7 promoter was removed by digestion with $B g l \mathrm{II}$ and NcoI enzymes and replaced by the TRC promoter obtained from pTRC99a plasmid by amplification and digestion with $\mathrm{BamHI}$ and $\mathrm{NcoI}$ to obtain the pTRCGQ plasmid. The empty plasmid (pCM) was constructed by incorporating the TRC promoter into the pET15c plasmid.

\section{Inactivation of gluQ-rs gene in S. flexneri}

Deletion of gluQ-rs was carried out using the $\lambda$ red recombinase method [44] with the following modifications. S. flexneri 2457T carrying pKD46 and prepared as described elsewhere [44] was transformed with a purified PCR fragment amplified from the E. coli $\Delta g l u Q-r s:: k a n$ mutant strain using primers dksAF and pcnBR (Table 2), increasing the homologous DNA region to more than $450 \mathrm{bp}$ at each side. The mutant was isolated following overnight growth at $37^{\circ} \mathrm{C}$ on LB-agar containing kanamycin $(50 \mu \mathrm{g} / \mathrm{ml})$. The deletion was confirmed by PCR using the same pair of primers (dksAF-pcnBR) and using each primer together with an internal primer as described previously [44]. The presence of the S. flexneri virulence plasmid was also confirmed by PCR amplification of the virF gene using primers virFF and virFR (Table 2).

\section{Effect of the absence of gluQ-rs gene in S. flexneri metabolism}

The effect of the deletion of the gluQ-rs gene on the metabolism of S. flexneri was analyzed by Biolog phenotype MicroArrays following the manufacturer's instructions (Biolog, Inc., Almeda, CA). Strains were grown at $30^{\circ} \mathrm{C}$ overnight and $5 \mathrm{ml}$ of LB was inoculated with a 1:100 dilution and grown at $37^{\circ} \mathrm{C}$ to reach an $\mathrm{OD}_{650 \mathrm{~nm}}$ of 0.5 . The cells were then washed and resuspended to $2.5 \times 10^{7} \mathrm{cfu} /$ $\mathrm{ml}$ and diluted 200 fold in to a solution of IF-10a medium (Biolog). Each well was inoculated with $1.2 \times 10^{4} \mathrm{cfu}$ (0.1 $\mathrm{ml}$ per well) into the corresponding plates and incubated for $24 \mathrm{hrs}$ at $37^{\circ} \mathrm{C}$. The metabolism was recorded and analyzed by the Omnilog software (V 1.20.02) (Biolog, Inc., Almeda, CA).

\begin{abstract}
Abbreviations
GluQ-RS: Glutamyl queuosine-tRNA ${ }^{\text {Asp }}$ synthetase and its codifying gene; tRNA ${ }^{\text {Asp: }}$ Transfer ribonucleic acid codifying aspartic acid; GluQ: Glutamylqueuosine; aaRS: Aminoacyl-tRNA synthetase; GluRS: Glutamyl-tRNA synthetase; Q: Queuosine; SPI-1: Pathogenicity islands of Salmonella; miaA: tRNA $\Delta$ (2)-isopentenylpyrophosphate transferase; tgt: tRNA guanine transglycosylase; RT-PCR: Reverse transcription-polymerase chain reaction; LB: Luria Bertani; MOPS: 3-(N-morpholino)propanesulfonic acid; MMuLV: Moloney Murine Leukemia Virus Reverse Transcriptase; PVDF: Polyvinyl difluoride.
\end{abstract}

\section{Competing interests}

The authors declare that they have no competing interests.

\section{Authors' contributions}

VCC: performed cloning, the enzymatic assay, data analysis. VPT: performed ex vivo assays of the wild type and mutant strains of Shigella and the enzymatic activity under different growth conditions. CM: performed the RT-PCR of the mRNA isolated from Shigella flexneri. CRF: performed the Biolog assay in collaboration with JCS. JCS: Analysis of the data, experimental design, N terminal determination of GluQ-RS. SMP and JCS: analyzed the 
results and wrote the paper. All authors contributed to the editing and approved the final paper.

\section{Acknowledgements}

We are grateful to Dr. Dieter Söll from Yale University, USA, for providing the E. coli strains BL21(DE3) and W3110 $\Delta$ gluQ-rs.:kan. Also, we would like to thank to Dr. Claude Parsot from the Institute Pasteur, France, for providing the PQF50 plasmid and advice in the determination of the N-terminal sequence of GluQ-RS. We appreciate Dr. Elizabeth Wyckoff for her critical review of this manuscript. This publication was funded by Grants from the Department of Research, University of Chile DI 12 06/04-2 and Fondo Nacional de Desarrollo Científico y Tecnólogico (FONDECYT) 1080308 to J.C. S. and Grant Al 169351 from the National Institutes of Health to S.M.P.

\section{Author details}

${ }^{1}$ Program of Microbiology and Mycology, Institute of Biomedical Science (ICBM), Faculty of Medicine, University of Chile, Santiago, Chile. ${ }^{2}$ Area of Biochemistry, Faculty of Dentistry, University of Chile, Santiago, Chile. ${ }^{3}$ Molecular Genetics and Microbiology, The University of Texas at Austin, Austin, TX, USA. ${ }^{4}$ Present address: Department of Molecular Genetics and Microbiology, Faculty of Biological Science, Catholic University, Santiago, Chile.

Received: 11 June 2012 Accepted: 11 September 2012 Published: 5 October 2012

\section{References}

1. Ibba M, Söll D: Aminoacyl-tRNA synthesis. Annu Rev Biochem 2000, 69:617-650.

2. Eriani G, Delarue M, Poch O, Gangloff J, Moras D: Partition of tRNA synthetases into two classes based on mutually exclusive sets of sequence motifs. Nature 1990, 347:203-206.

3. Woese CR, Olsen GJ, Ibba M, Söll D: Aminoacyl-tRNA synthetases, the genetic code, and the evolutionary process. Microbiol Mol Biol Rev 2000, 64:202-236

4. Skouloubris $\mathrm{S}$, de Pouplana $L R$, de Reuse $H$, Hendrickson $H$ : A noncognate aminoacyl-tRNA synthetase that may resolve a missing link in protein evolution. Proc Natl Acad Sci USA 2003, 100:11297-11302

5. Salazar JC, Ahel I, Orellana O, Tumbula-Hansen D, Krieger R, Daniels L, Söll D: Coevolution of an aminoacyl-tRNA synthetase with its tRNA substrates. Proc Natl Acad Sci USA 2003, 100:13863-13868.

6. Schimmel P, Ripmaster T: Modular design of components of the operational RNA code for alanine in evolution. Trends Biochem Sci 1995, 20:333-334

7. Sissler M, Delorme C, Bond J, Ehrlich SD, Renault P, Francklyn C: An aminoacyl-tRNA synthetase paralog with a catalytic role in histidine biosynthesis. Proc Natl Acad Sci USA 1999, 96:8985-8990.

8. Navarre WW, Zou SB, Roy H, Xie JL, Savchenko A, Singer A, Edvokimova E, Prost LR, Kumar R, Ibba M, Fang FC: PoxA, YjeK, and elongation factor $P$ coordinately modulate virulence and drug resistance in Salmonella enterica. Mol Cell 2010, 39:209-221.

9. Bearson SM, Bearson BL, Brunelle BW, Sharma VK, Lee IS: A mutation in the poxA gene of Salmonella enterica serovar Typhimurium alters protein production, elevates susceptibility to environmental challenges, and decreases swine colonization. Foodborne Pathog Dis 2011, 8:725-732.

10. Salazar JC, Ambrogelly A, Crain PF, McCloskey JA, Söll D: A truncated aminoacyl-tRNA synthetase modifies RNA. Proc Natl Acad Sci USA 2004, 101:7536-7541.

11. Dubois DY, Blaise M, Becker HD, Campanacci V, Keith G, Giegé R, Cambillau C, Lapointe J, Kern D: An aminoacyl-tRNA synthetase-like protein encoded by the Escherichia coli yadB gene glutamylates specifically

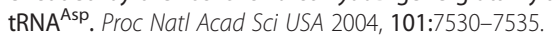

12. Iwata-Reuyl D: Biosynthesis of the 7-deazaguanosine hypermodified nucleosides of transfer RNA. Bioorg Chem 2003, 31:24-43.

13. Gustilo EM, Vendeix FA, Agris PF: tRNA's modifications bring order to gene expression. Curr Opin Microbiol 2008, 11:134-140.

14. Morris RC, Brown KG, Elliott MS: The effect of queuosine on tRNA structure and function. J Biomol Struct Dyn 1999, 4:757-77414.

15. Harada F, Nishimura S: Possible anticodon sequences of tRNA ${ }^{\text {His }}, t_{R N A}{ }^{A s n}$ and tRNA ${ }^{\text {Asp }}$ from Escherichia coli B. Universal presence of nucleoside Q in the first position of the anticodons of these transfer ribonucleic acids. Biochem 1972, 11:301-308.

16. Durand JM, Okada N, Tobe T, Watarai M, Fukuda I, Suzuki T, Nakata N, Komatsu K, Yoshikawa M, Sasakawa C: vacC, a virulence-associated chromosomal locus of Shigella flexneri, is homologous to tgt, a gene encoding tRNA-guanine transglycosylase (Tgt) of Escherichia coli K-12. J Bacteriol 1994, 176:4627-4634.

17. Durand JM, Björk GR, Kuwae A, Yoshikawa M, Sasakawa C: The modified nucleoside 2-methylthio-N6-isopentenyladenosine in tRNA of Shigella flexneri is required for expression of virulence genes. J Bacteriol 1997, 179:5777-5782.

18. Urbonaviĉius J, Qian Q, Durand JM, Hagervall TG, Björk GR: Improvement of reading frame maintenance is a common function for several tRNA modifications. EMBO J 2001, 20:4863-4873.

19. Szklarczyk D, Franceschini A, Kuhn M, Simonovic M, Roth A, Minguez $P$, Doerks T, Stark M, Muller J, Bork P, Jensen LJ, Mering C: The STRING database in 2011: functional interaction networks of proteins, globally integrated and scored. Nucleic Acids Res 2011, 39:D561-D568.

20. Kanehisa M: Linking databases and organisms: GenomeNet resources in Japan. TIBS 1997, 22:442-444.

21. Tamura K, Peterson D, Peterson N, Stecher G, Nei M, Kumar S: MEGA5: molecular evolutionary genetics analysis using maximum likelihood, evolutionary distance, and maximum parsimony methods. Mol Biol Evol 2011, 28:2731-2739.

22. Kang PJ, Craig EA: Identification and characterization of a new Escherichia coli gene that is a dosage-dependent suppressor of a dnaK deletion mutation. J Bacteriol 1990, 172:2055-2064.

23. Farinha MA, Kropinski AM: Construction of broad-host-range plasmid vectors for easy visible selection and analysis of promoters. J Bacteriol 1990, 172:3496-3499.

24. Sambrook J, Fritsch EF, Maniatis T: Molecular Cloning: A Laboratory Manual. 2nd edition. USA: Cold Spring Harbor Laboratory Press; 1989.

25. Chandrangsu P, Lemke JJ, Gourse RL: The $d k s A$ promoter is negatively feedback regulated by DksA and ppGpp. Mol Microbiol 2011, 80:1337-1348.

26. Zuker M: Mfold web server for nucleic acid folding and hybridization prediction. Nucleic Acids Res 2003, 31:3406-3415.

27. Mogull SA, Runyen-Janecky $L$, Hong M, Payne SM: $d k s A$ is required for intercellular spread of Shigella flexneri via an RpoS-independent mechanism. Infect Immun 2001, 69:5742-5751.

28. Sharma UK, Chatterji D: Transcriptional switching in Escherichia coli during stress and starvation by modulation of sigma activity. FEMS Microbiol Rev 2010, 34:646-657.

29. Du H, Wang M, Luo Z, Ni B, Wang F, Meng Y, Xu S, Huang X: Coregulation of gene expression by sigma factors RpoE and RpoS in Salmonella enterica serovar Typhi during hyperosmotic stress. Curr Microbiol 2011 62:1483-1489.

30. Durfee T, Hansen A-M, Zhi H, Blattner FR, Jin DJ: Transcription profiling of the stringent response in Escherichia coli. J Bacteriol 2008, 190:1084-1096.

31. Booth IR, Higgins CF: Enteric bacteria and osmotic stress: intracellular potassium glutamate as a secondary signal of osmotic stress? FEMS Microbiol Rev 1990, 6:239-246.

32. Golovina AY, Sergiev PV, Golovin AV, Serebryakova MV, Demina I, Govoru VM, Dontsova OA: The yfiC gene of $E$. coli encodes an adenine-N6 methyltransferase that specifically modifies $\mathrm{A} 37$ of $\mathrm{tRNA}_{1}^{\text {Val }}\left(\mathrm{cmo}^{5} \mathrm{UAC}\right)$. RNA 2009, 15:1134-1141.

33. Smiley BL, Lupski JR, Svec PS, McMacken R, Godson GN: Sequences of the Escherichia coli dnaG primase gene and regulation of its expression. Proc Natl Acad Sci USA 1982, 79:4550-4554.

34. Pagès V, Koffel-Schwartz N, Fuchs RPP: recX, a new SOS gene that is co-transcribed with the recA gene in Escherichia coli. DNA Repair 2003, 2:273-284.

35. Garst AD, Edwards AL, Batey RT: Riboswitches: structures and mechanisms. Cold Spring Harbor Perspect Biol 2011, 3:a003533.

36. Roth A, Winkler WC, Regulski EE, Lee BWK, Lim J, Jona I, Barrick JE, Ritwik A, Kim JN, Welz R, Iwata-Reuyl D, Breaker RR: A riboswitch selective for the queuosine precursor preQ1 contains an unusually small aptamer domain. Nat Struct Mol Biol 2007, 14:308-317.

37. Chang TH, Huang HD, Wu LC, Yeh CT, Liu BJ, Horng JT: Computational identification of riboswitches based on RNA conserved functional sequences and conformations. RNA 2009, 15:1426-1430. 
38. Fisher CR, Davies NM, Wyckoff EE, Feng Z, Oaks EV, Payne SM: Genetics and virulence association of the Shigella flexneri sit iron transport system. Infect Immun 2009, 77:1992-1999.

39. Edgar RC: MUSCLE: multiple sequence alignment with high accuracy and high throughput. Nucleic Acids Res 2004, 32:1792-1797.

40. Yu Z, Morrison M: Comparisons of different hypervariable regions of $r$ rs genes for use if fingerprinting of microbial communities by PCRDenaturing Gel Electrophoresis. Appl Environ Microbiol 2004, 70:4800-4806.

41. Vidal M, Kruger E, Durán C, Lagos R, Levine M, Prado V, Toro C, Vidal R: Single multiplex PCR assay to identify simultaneously the six categories of diarrheagenic Escherichia coli associated with enteric infections. J Clin Microbiol 2005, 43:5362-5365.

42. Miller J: Experiments in Molecular Genetics. NY: Cold Spring Harbor Laboratory; 1972:352-355.

43. Ho SN, Hunt HD, Horton RM, Pullen JK, Pease LR: Site-directed mutagenesis by overlap extension using the polymerase chain reaction. Gene 1989, 77:51-59.

44. Datsenko KA, Wanner BL: One-step inactivation of chromosomal genes in Escherichia coli K-12 using PCR products. Proc Natl Acad Sci USA 2000, 97:6640-6645.

doi:10.1186/1471-2180-12-226

Cite this article as: Caballero et al:: Expression of Shigella flexneri gluQ-rs gene is linked to $d k s A$ and controlled by a transcriptional terminator. BMC Microbiology 2012 12:226.

\section{Submit your next manuscript to BioMed Central and take full advantage of:}

- Convenient online submission

- Thorough peer review

- No space constraints or color figure charges

- Immediate publication on acceptance

- Inclusion in PubMed, CAS, Scopus and Google Scholar

- Research which is freely available for redistribution 